\title{
Ionic migration behavior in minute wiring on flexible substrate
}

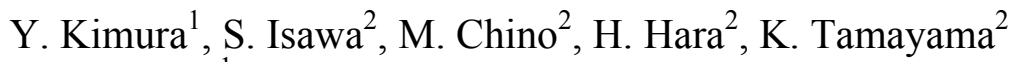 \\ \& A. Suzuki ${ }^{1}$ \\ ${ }^{I}$ Department of Materials Science and Technology, Kogakuin University, \\ Shinjuku-ku, Tokyo, Japan \\ ${ }^{2}$ MISUZU Industrial Corporation, Nakaminowa, Minowacho, \\ Kamiinagunn, Nagano, Japan
}

\begin{abstract}
Recently, miniaturization and high-density mounting have been demanded in various electronic devices. Above all, flexible print circuit (FPC) technology has been a focal point, especially in the field of notebook computers and cellular phones, for obtaining a higher degree of freedom in design. In order to actualize the fine pitch of wiring in FPC, the insulation degradation due to the migration becomes a problem that cannot be ignored. Because of differences in its structure and wiring from the former printed circuit board, the analysis of migration behavior in FPC becomes important for evaluating the reliability of an electronic component based on FPC. In this research, in order to grasp the migration behavior of FPC, a $45 \mu \mathrm{m}$ pitched COF specimen was used. In addition, the paragraphed $30 \mu \mathrm{m}$ pitch tooth profile wiring specimen was designated as the test material. Then, a reliability test for evaluating the migration behavior was conducted. As a result of a Steady-State Temperature Humidity Bias Life (THB) test, the migration occurred progressively. Various investigations were conducted for explaining about the mechanism of ionic migration. Also in this paper, the adhesive strength between the polyimide film and copper $(\mathrm{Cu})$ wiring pattern, and that between the polyimide film and the under-filling material, was measured in order to investigate the influence of the surface state of polyimide film upon the migration behavior.
\end{abstract}

Keywords: ionic migration, FPC, reliability, THB testing. 


\section{Introduction}

Recently, miniaturization and high-density mounting have been demanded in various electronic devices. Above all, flexible print circuit has been a focal point especially in the field of notebook computers and cellular phones for realizing high density mounting and also obtaining a higher degree of freedom in design. The insulation failure, due to an ionic migration damage [1,2] with an advanced fine pitch wire mounting, becomes a serious problem [3]. Ionic migration is an electrochemical phenomenon related to chemical solutions under electric potential. The reaction mechanisms of ionic migration can be classified into three processes: anodic dissolution, mass transfer, and cathodic deposition [4]. Migration in electronic materials is caused by an electrochemical phenomena related to chemical solutions and electric potential $[5,6]$. Deposition reactions and metal ion transfer reactions are involved in migration, induced by such factors as metal dissolution reactions and diffusion [7,8]. Migration is especially likely to occur when electronic devices using high-density mounting are affected by both materials and environmental conditions [9]. When migration occurs, it causes changes in electrical characteristics and results in equipment failure.

"Migration" refers to a phenomenon in which metal ions are transferred from one metal electrode to the opposite metal electrode, this process results in metal or alloy deposits. The transfer occurs between the electrodes of devices such as printed circuit boards (PCBs) when an electric field is impressed in the presence of moisture such as dew condensation adhering between the electrodes. Migration is classified as either dendrite or conductive anodic filament depending on the shape of the deposits and the conditions leading to the occurrence $[1,10]$ "Dendrite" refers to dendritic-shaped metal or metal oxide deposits on the surface of PCB insulation.

The analysis of migration behaviour in FPC becomes important for evaluating the reliability of electronic devices based on FPC. In this research, THB tests under the conditions of $85^{\circ} \mathrm{C}$ and $85 \% \mathrm{RH}$, with applying electric potentials of 5 , 25 and $45 \mathrm{v}$, were conducted using COF as a sample to understand the ionic migration behaviour in FPC. Various investigations concerning generated ionic migration behaviour were conducted employing SEM and EDX. Some trials were conducted for establishing the evaluation method of ionic migration behaviour. In addition, improvement of ionic migration character was conducted employing alternative under fill resin in FPC materials system.

\section{Experimental procedures}

\subsection{Specimen and THB accelerated migration test}

COF structure of the test specimen is shown in detail in fig. 1. The specimen used in this study is that composed of polyimide substrate and consisted of an $8 \mu \mathrm{m}$ thick $\mathrm{Cu}$ wiring that was covered with $0.2 \mu \mathrm{m}$ thick plated tin $(\mathrm{Sn})$, coating as shown in fig. 1 (c). IC, whose size is $1.96 \mathrm{~mm} \times 19 \mathrm{~mm}$, is mounted on Sn plated $\mathrm{Cu}$ wiring on FPC using gold $(\mathrm{Au})$ bump with a thickness of $14 \mu \mathrm{m}$. 
Epoxy resin was used for an under fill material and polyimide resin was used as a solder resistant of FPC. The COF specimen used in this study was prepared using $\mathrm{Cu}$ wiring plated on a $\mathrm{Cr} / \mathrm{Ni}$ seed layer sputtered on polyimide resin substrate.

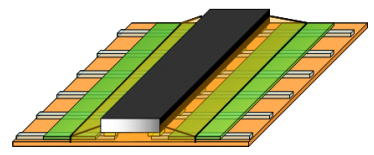

(a) Outlook of COF

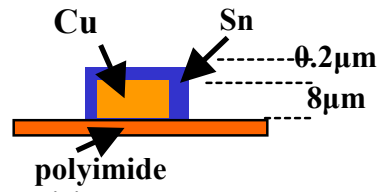

(b) $\mathrm{Cu}$ wiring geometry

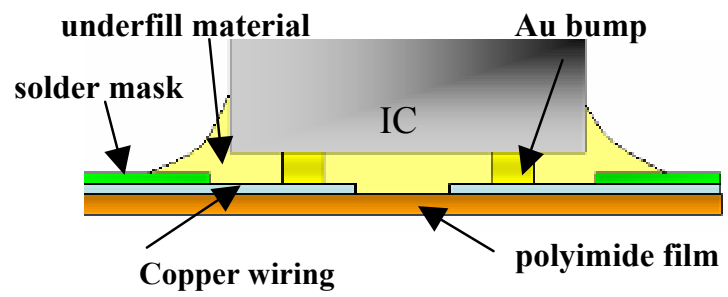

(c) Detailed structure of COF

Figure 1: COF structure of test specimen: $45 \mu \mathrm{m}$ pitch.

Employing this COF sample with a $45 \mu \mathrm{m}$ pitch, an accelerated migration test was conducted up to $168 \mathrm{hrs}$ long employing a THB test. The conditions of the THB test were $85^{\circ} \mathrm{C}$ and $85 \% \mathrm{RH}$ whilst applying electric potentials of 5, 25 and $45 \mathrm{v}$.

\subsection{Observation of migration morphology and leak test result}

After conducting THB tests of 24, 70, 96 and 168 hrs, morphology change concerning migration generation in test specimens was examined by using an optical microscope. At the same time, a leak test was conducted for each specimen under a constant condition.

\subsection{SEM and EDX evaluation of migration behaviour}

Morphologies of migration damage and the generated dendrite structures were investigated by SEM. Characterization of generated materials observed on a cross section of a specimen, with THB accelerated migration test, was conducted employing EDX.

\section{Experimental results and discussions}

\subsection{Result of THB accelerated migration test}

THB tested specimens under the condition of $85^{\circ} \mathrm{C}$ and $85 \% \mathrm{RH}$ showed insulation failure for the first time after $168 \mathrm{hrs}$. Color change was recognized in 
degraded specimens on the interface between the $\mathrm{Cu}$ line and polyimide substrate after $24 \mathrm{hrs}$ of THB testing. Also dendrite was generated from the side part of Sn coated $\mathrm{Cu}$ wiring, which was anode. These degradations (shown in fig. 3) were mainly recognized in the areas from $A$ to $\mathrm{C}$ originally indicated in fig. 2, which were covered with under fill material. Therefore, under fill material may affect migration behaviour of this specimen.
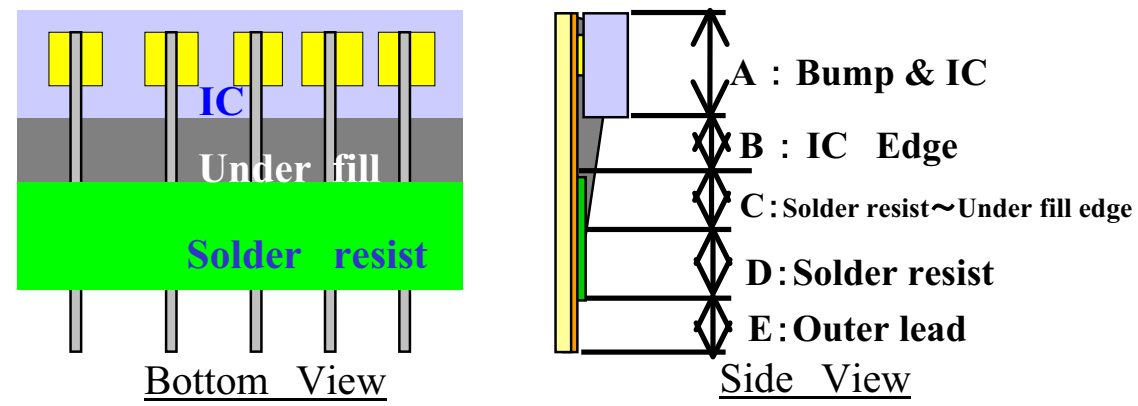

Figure 2: $\quad$ Observed sample parts.
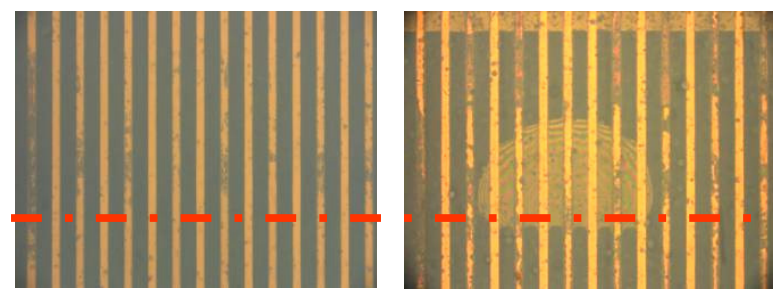

B

(a) after $24 \mathrm{hrs}$ THB testing (b) after $168 \mathrm{hrs}$ THB testing

Figure 3: Bottom view of migration damage observed by an optical microscope mainly generated in $\mathrm{B}$ and $\mathrm{C}$ regions shown in fig. 2.

Migration behaviour generated in COF can be characterized by the color change due to the dissolution of the $\mathrm{Cu}$ lines and dendritic precipitation of $\mathrm{Cu}$. These electrochemical processes may result in the insulation failure of FPC. The generation of migration with time, under THB testing conditions of $85^{\circ} \mathrm{C}$ and $85 \%$ RH was investigated and summarized in Table 1 . As a result, color change occurred after $24 \mathrm{hrs}$ testing due to the dissolution of $\mathrm{Cu}$ lines and dendritic precipitation of $\mathrm{Cu}$. On the contrary, insulation failure was detected for the first time only after $168 \mathrm{hrs}$ THB testing. Therefore, the migration process was proceeded from the initial stage of THB testing at which insulation failure was not detected. 

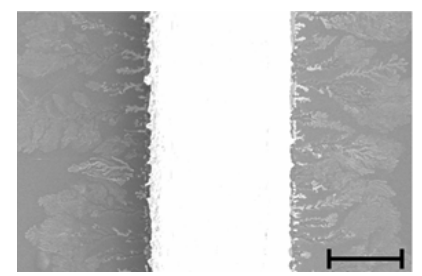

(a) Surface view of wiring

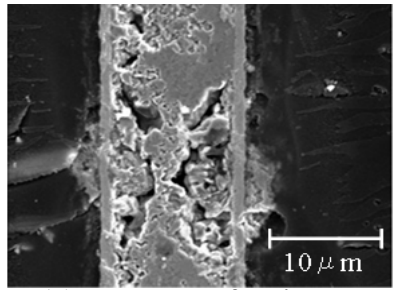

(c) Bottom of wring

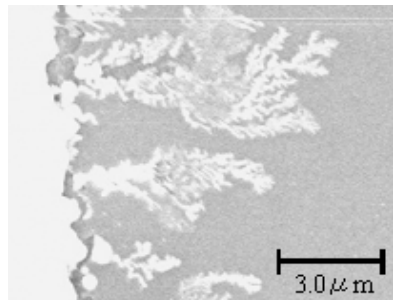

(b) Close up of dendrite

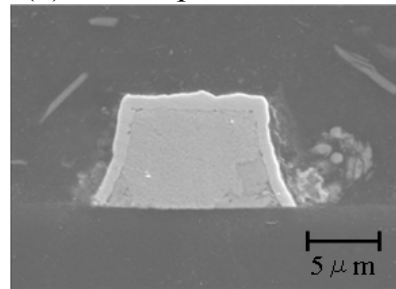

(d) Cross-section of wiring

Figure 4: Migration observed by SEM.

Table 1: Generation of migration with time.

\begin{tabular}{|c|l|l|c|}
\hline \multirow{2}{*}{ Time } & \multicolumn{2}{|c|}{ Outlook } & \multirow{2}{*}{ Impedance } \\
\cline { 2 - 3 } & Color change & Dendrite & Good \\
\hline 24 & Yes & Yes & Good \\
\hline 70 & Yes & Yes & Good \\
\hline 96 & Yes & Yes & Degraded \\
\hline 168 & Yes & Yes & \\
\hline
\end{tabular}

\subsection{Migration behaviour detected by SEM and EDX}

The generated morphology of migration was indicated in fig. 4. Surface morphology of wiring, after generating migration and close up features of dendrite, were examined by SEM and indicated in fig. 4 (a) and (b). Damaged morphologies on the bottom and the cross-section of wiring are shown in fig. 4 (c) and (d). Therefore dendrite formation was essential for the migration process. Dissolved morphology was recognized on the bottom of $\mathrm{Cu}$ wiring; however, $\mathrm{Sn}$ plated coating was left undamaged. Cross-sectional view of wiring shown in fig. 4(d) indicates that migration damage was initiated from interfacial region between Sn plated coating and polyimide substrate. Then the central parts of the $\mathrm{Cu}$ line bottom were dissolved out with the progress of migration damage.

The results of the EDX analysis for the cross-sectional area of wiring are shown in fig. 5. This figure indicates that $\mathrm{Cu}$ was dissolved from the line via an interface between plated $\mathrm{Sn}$ coating and polyimide substrate. From these observations, elements of dendrite were composed of $\mathrm{Cu}$ dissolved from lines and precipitated in the under fill resin. Also, in EDX mapping shown in fig. 5, 
the chlorine's $(\mathrm{Cl})$ presence peaked in surrounding areas around $\mathrm{Sn}$ plated $\mathrm{Cu}$ wiring, which was included in under fill resin. Then, morphology of dendrite was investigated in detail and shown in fig. 6. As a result, initiation of dendrite formation was not recognized in the vicinity of Sn plating, however, dendrite formation was initiated from some distant area from Sn plating. Judging from this figure and observed results obtained from specimens before THB testing, the initiation site of dendrite was understood to be the $\mathrm{Cr} / \mathrm{Ni}$ seed layer which is an essential element for making specimens by sputtering and plating process.

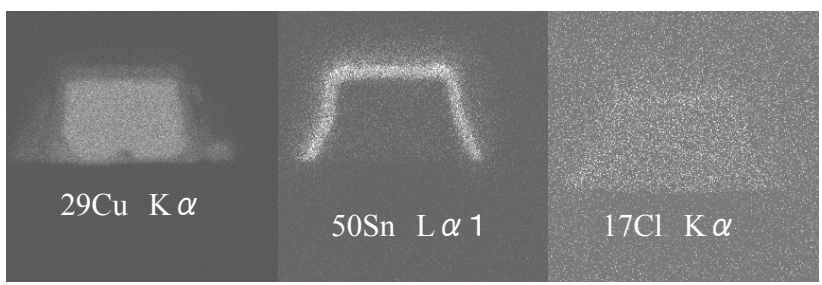

Figure 5: Analysis of migrated element by EDX.

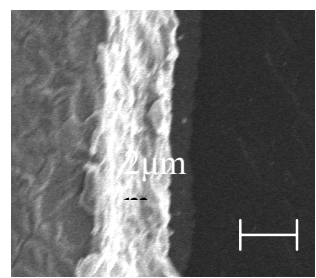

(a) $\mathrm{Cr} / \mathrm{Ni}$ seed layer

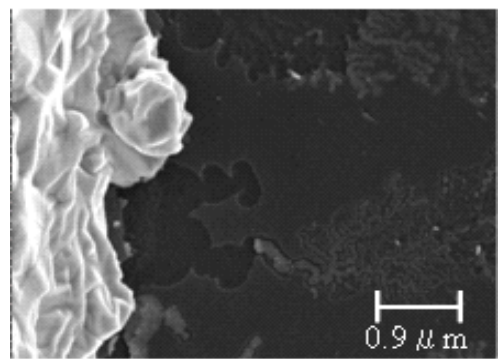

(b) Dendrite generation

Figure 6: $\quad$ Observation of initial migration site by SEM.

Table 2: $\quad$ Comparison of elements of under fill material.

\begin{tabular}{c|c|c}
\hline & Usual under fill & Improved under fill \\
\hline Main element & Epoxy & Epoxy \\
$\mathrm{Cl}^{-}$ion $(\mathrm{ppm})$ & 0.41 & 0.12 \\
$\mathrm{Na}^{+}$ion $(\mathrm{ppm})$ & 0.32 & 0.15 \\
$\mathrm{pH}$ & 5 & 4.1 \\
Conductivity $(\mu \mathrm{S} / \mathrm{cm})$ & 21.2 & 27.7 \\
Water absorption $(\mathrm{wt} . \%)$ & $1 \sim 2$ & 1.4 \\
Viscosity $(\mathrm{Pa} \cdot \mathrm{s})$ & 0.45 & 19 \\
\hline
\end{tabular}




\subsection{Improvement of migration resistance by applying alternative under fill resin}

The EDX result shown in fig. 5 indicated that $\mathrm{Cl}$ content of under fill resin might significantly affect migration behaviour. Therefore, some trials for improving migration resistance were conducted employing alternative resin whose content of $\mathrm{Cl}$ was suppressed. Chemical compositions of both usual and alternative under fill resin were indicated in Table 2. Another type of COF specimen whose dimensions were the same as shown in fig. 1, except for under fill material, was prepared. THB testing under the same condition was then conducted employing this type of specimen. After $168 \mathrm{hrs}$ THB testing, good results were obtained, both in outlook evaluation and impedance testing, for this type of specimen with alternative under fill material system.

\subsection{Adhesive strength degradation after THB testing}

SEM/EDX analysis indicated that migration was initiated from an interface between the substrate and plated Sn coating. Therefore, the adhesive strength of the interface between the substrate and coating may affect the migration behaviour of COF. Evaluation of degradation characteristics of adhesive strength due to THB testing is extremely important. In this chapter, comparison of adhesive strength before and after THB testing was conducted. Also in this research, the difference in under fill resin was taken into consideration. The peeling strength of under fill resin from substrate was measured and the test results are shown in fig. 7 . The initial peeling strength was different depending upon the type of resin. Alternative under fill material that suppressed $\mathrm{Cl}$ content showed improved adhesive strength compared with the usual type of resin.

Degradation in peeling strength was detected in both usual and improved under fill resin; however, degraded ratio in peeling strength is obtained to be better for improved resin. The value of the peeling load for improved resin, after THB testing, remained at about $80 \%$ of the usual resin before THB testing. Therefore, the advantage of improved resin was indicated from the viewpoint of peeling strength degradation by THB testing.

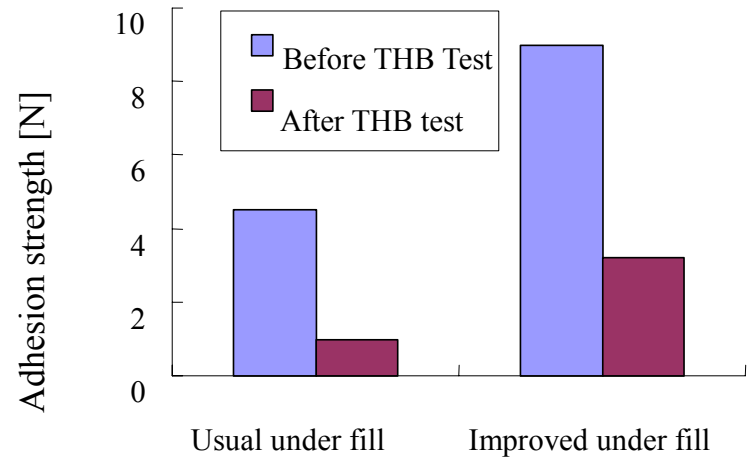

Figure 7: Comparison of adhesion strength before and after 168 hrs THB test. 


\subsection{Dependency of migration behavior of FPC upon manufacturing process and under fill resin}

In this section, effects of manufacturing methods and under fill resin upon the migration behavior of FPC were evaluated employing a $30 \mu \mathrm{m}$ pitched tooth profile wiring specimen shown in fig. 8 .

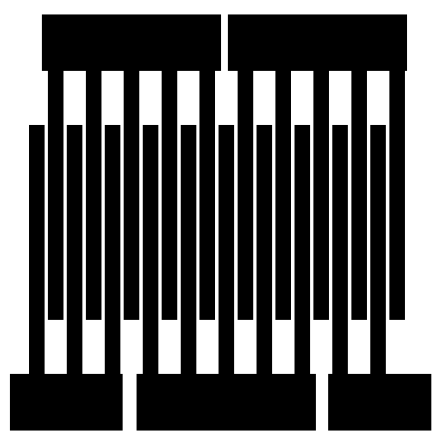

(a) Enlarged view of tooth profile wiring
Under fill resin

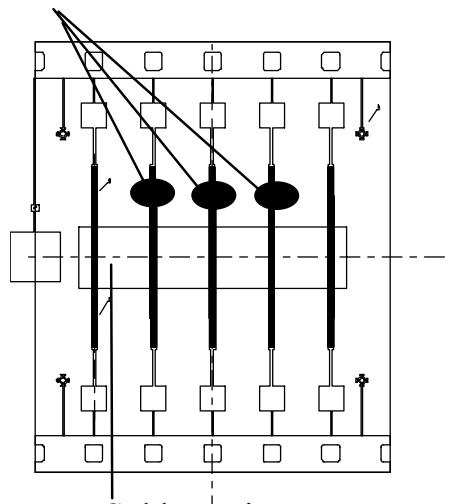

Solder resist

(b) Outlook of tooth profile wiring specimen

Figure 8: $\quad$ FPC tooth profile wiring specimen with $30 \mu \mathrm{m}$ pitch.

Table 3: $\quad$ Combination of under fill resin and substrate.

\begin{tabular}{|c|c|c|}
\hline Under fill resin type & $\begin{array}{c}\text { Substrate: a } \\
\text { [Casting method] }\end{array}$ & $\begin{array}{c}\text { Substrate: b } \\
\text { [Cr/Ni Sputtering \& } \\
\text { Cu electroplating] }\end{array}$ \\
\hline Resin A ( Epoxy) & Sample A-a & Sample A-b \\
\hline Resin B ( Epoxy) & Sample B-a & Sample B-b \\
\hline Resin C ( Epoxy) & Sample C-a & Sample C-b \\
\hline Resin D ( Epoxy) & Sample D-a & Sample D-b \\
\hline Resin E ( Epoxy) & Sample E-a & Sample E-b \\
\hline Non & Sample 0-a & Sample 0-b \\
\hline
\end{tabular}


In this paper, both the sputtering \& electroplating (substrate: b) and casting method (substrate: b) were employed for manufacturing FPC. On polyimide substrate, about $40 \mu \mathrm{m}$ thick, $8 \mu \mathrm{m}$ of $\mathrm{Cu}$ line, with $0.2 \mu \mathrm{m}$ of Sn plating, was formed. Polyimide resin was also used for solder resistant and 5 under fill resin types (A, B, C, D and E) of epoxy resins were applied. A specimen without under fill resin was also used. On some part of the tooth profile wiring specimen shown in fig. 8 (b), under fill resin was applied and hardened under adequate curing conditions. Specimens used in this study are summarized in Table 3. THB testing was employed in these specimens by measuring the impedance of them with time. Test conditions were the same as the previous test, that is, $85^{\circ} \mathrm{C}, 85 \%$ $\mathrm{RH}$ and a bias potential of DC45V.

After $200 \mathrm{hrs}$ of THB testing had been conducted, an optical microscope detected the outlook of the specimen. As a result, some color change was detected in some part of specimens using substrate:a, however no dendrite formation was recognized in these types of specimens. On the contrary, in the case of specimens using substrate:b, color change on wiring and also dendrite formation were clearly recognized. Color change and dendrite formation were initiated from the boundary layer between wiring and polyimide substrate. These degradations were generated only from the anode part of wiring as shown in fig. 9. These results almost corresponded with the results indicated in previous sections that were obtained from the test specimen shown in fig. 1.

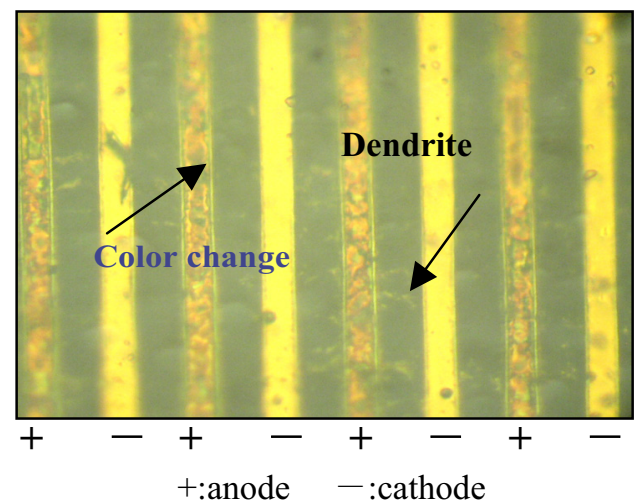

Figure 9: Dendrite formation after 200hrs THB testing. Sample E-b.

From the above mentioned result, color change and dendrite formation behavior have a dependency on under fill resin and manufacturing processes. Therefore in the following process, dependency of migration behavior on FPC upon under fill resin and substrate material was examined through evaluating impedance change with THB testing time. Insulation degradation with THB testing, detected by impedance change, is summarized in Table 4.

Judging from the impedance value of the specimen, using substrate:a showed better ionic migration resistance. Impedance values have been kept greater than $100 \mathrm{M} \Omega$ for three kinds of under fill resins: A, B \& E, and the value was kept 
greater than $1 \mathrm{M} \Omega$ for two kinds of under fill resin: C \& D in this case. In the case of specimens using substrate:b, no specimen kept its impedance value grater than $100 \mathrm{M} \Omega$ after $200 \mathrm{hrs}$ of THB testing. Only one kind of specimen with under fill resin $\mathrm{D}$ and the non-resin coated specimen managed to keep a good impedance condition ( $\mathrm{I} \geqq 1 \mathrm{M} \Omega$ ) after $200 \mathrm{hrs}$ THB testing. The other four kinds of under fill resin coated specimens showed insulation degradation within $10 \mathrm{hrs}$. From these test results, it was clarified that specimens using substrate:a showed better insulation degradation resistance compared with those using substrate:b.

Table 4: $\quad$ Insulation degradation detected by impedance change.

\begin{tabular}{|c|c|c|}
\hline & Substrate a & Substrate b \\
\hline Resin A & Sample A-a & Sample A-b \\
\hline Time to insulation degradation & (0) & $\times: 4.4 \mathrm{hrs}$ \\
\hline Resin B & Sample B-a & Sample B-b $\times$ \\
\hline Time to insulation degradation & () & $8.6 \mathrm{hrs}$ \\
\hline Resin C & Sample C-a & Sample C-b \\
\hline Time to insulation degradation & $\bigcirc$ & $\times: 5.8 \mathrm{hrs}$ \\
\hline Resin D & Sample D-a & Sample D-b \\
\hline Time to insulation degradation & $\bigcirc$ & $\bigcirc$ \\
\hline Resin E & Sample 0-a & Sample 0-b \\
\hline Time to insulation degradation & () & $\times: 5.8 \mathrm{hrs}$ \\
\hline Non resin & 21 & 23 \\
\hline Time to insulation degradation & $\times: 161.8 \mathrm{hrs}$ & $\bigcirc$ \\
\hline & \multicolumn{2}{|c|}{$\begin{array}{l}(0): \text { Fairly good }(\mathrm{I} \geqq 100 \mathrm{M} \Omega) \\
\mathrm{O}: \operatorname{Good}(\mathrm{I} \geqq 1 \mathrm{M} \Omega) \\
\times: \text { Degraded }(\mathrm{I} \leqq 1 \mathrm{k} \Omega)\end{array}$} \\
\hline
\end{tabular}

For evaluating long-term reliability of specimens an additional $800 \mathrm{hrs}$ THB testing was conducted till 1,000 hrs was achieved through employing four kinds of specimens, that is, B-a, C-a. D-a and E-a, which showed no color change and kept good impedance value larger than $1 \mathrm{M} \Omega$ after $200 \mathrm{hrs}$ THB testing as shown in Table 4. Results obtained in this test are summarized in Table 5. No impedance degradation was detected in specimens B-a and C-a, however two other kinds of specimens were degraded at the stage of more than $800 \mathrm{hrs}$ THB testing as shown in Table 5.

In the specimens B-a and $\mathrm{C}-\mathrm{a}$, which showed no impedance degradation, some color change was detected as shown in fig. 10. In the specimens D-a and Ea, which showed impedance degradation before 1,000 hrs, remarkable color change was detected and is also shown in fig.10 (c) and (d). 
Table 5: $\quad$ Impedance change with THB test time till 1,000 hrs.

\begin{tabular}{|c|c|}
\hline Sample & I : Impedance \\
\hline B-a & Fairly good: $\bigcirc(\mathrm{I} \geqq 100 \mathrm{M} \Omega)$ \\
\hline C-a & Good : $\bigcirc(\mathrm{I} \geqq 1 \mathrm{M} \Omega)$ \\
\hline D-a & Degraded $\times(\mathrm{I} \leqq 1 \mathrm{k} \Omega)$ after $997.6 \mathrm{hrs}$ \\
\hline E-a & Degraded $: \times(\mathrm{I} \leqq 1 \mathrm{k} \Omega)$ after $810.8 \mathrm{hrs}$ \\
\hline
\end{tabular}

\section{Conclusions}

In this research, Steady-State Temperature Humidity Bias Life Test (THB Test) was conducted using $\mathrm{COF}$ as a sample to understand the ionic migration behaviour in FPC. Then, investigation concerning generated ionic migration behaviour was conducted employing SEM and EDX. In addition, some trials were conducted for establishing the evaluation method of ionic migration behaviour.

Results obtained are summarized as follows:

1. Migration behaviour such as color change that corresponded with dendrite formation was recognized in the specimen after $24 \mathrm{hrs}$ THB testing, however, insulation failure of specimen was detected after 168 hrs THB testing.

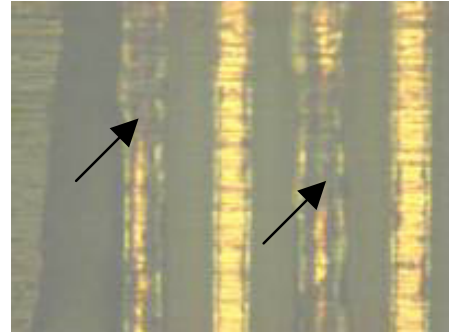

(a) Sample B-a

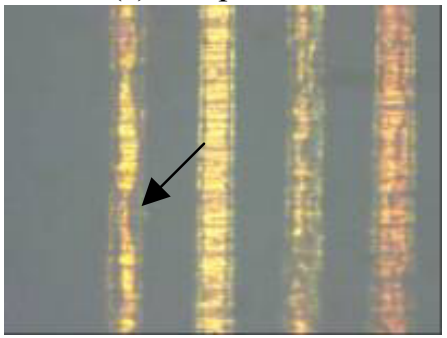

(c) Sample D-a

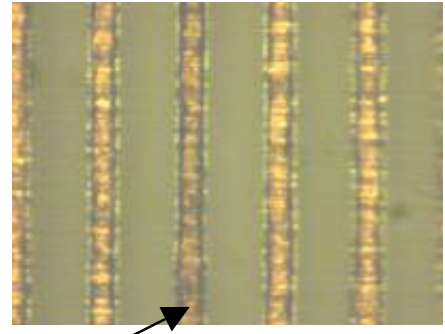

(b) Sample C-a

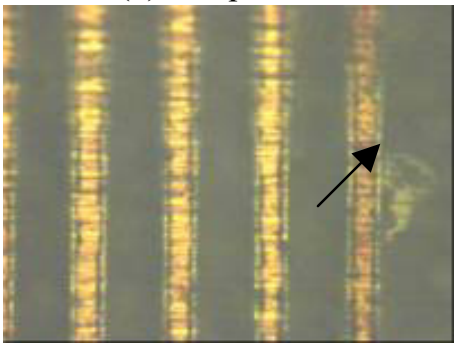

(d) Sample E-a

Figure 10: Outlook of specimen after additional 800 hrs (total 1,000 hrs) THB test. 
2. Dendrite generated from wiring on FPC was composed of $\mathrm{Cu}$, and this dendrite was initiated from the interface between plated $\mathrm{Sn}$ coating and polyimide substrate.

3. $\mathrm{Cl}$ included in epoxy resin used for under fill material is dominant factor affecting migration behaviour. Absorbed moisture in under fill resin, and the bias potential between anode and cathode electrodes, accelerated migration behaviour.

4. Generation of migration was initiated from some distant area due to Sn plating. The initiation site of dendrite was understood to be the $\mathrm{Cr} / \mathrm{Ni}$ seed layer, which is an essential element for making specimens by the sputtering and plating process.

5. Results of THB testing till $1,000 \mathrm{hrs}$, employing $30 \mu \mathrm{m}$ pitched tooth profile wiring specimen, showed that manufacturing method and types of under fill resin clearly affect migration behavior of FPC.

\section{References}

[1] G.T. Kohman, H.W. Hermance and G.H. Dowenes, "Silver Migration in Electrical Insulation” Bell System Tech. Journal, Vol.34, No.6, pp.1115$1147,1955$.

[2] S. Krumbein, "Metallic Electromigration Phenomena," IEEE Transactions on Components, Hybrids, and Manufacturing Technology, Vol.11, No.1, pp.5-15, 1988.

[3] Tsutomu Tsukui, "Insulation Deterioration and the Prevention Method by Electrochemical Migration of Electronic Equipment (Part 1)", The Journal of Japan Institute of Electronics Packaging, Vol.8, No.4, pp.339-345, 2005 .

[4] Hirokazu Tanaka, "Factors leading to ionic migration in lead-free solder", ESPEC Technology Report No. 14, pp. 1-9, 2002.

[5] M. Pourbaix, "Atlas of Electrochemical Equilibria in Aqueous Solutions", NACE, 1966.

[6] J.A. Augis, D.G. DeNure, M.J. LuValle, J.P. Mitchell, M.R. Pinnel and Welsher, T.L. "A Humidity Threshold for Conductive Anodic Filaments in Epoxy Glass Printed Wiring Boards", 3rd International SAMPE Electronics Conference, pp. 1023-1030, 1989.

[7] T.L. Welsher, J.P. Mitchell and D.J. Lando, "CAF in Composite PrintedCircuit Substrates: Characterization, Modeling, and a Resistant Material," Reliability Physics, 18th Annual Proceeding, pp 235-237, 1980.

[8] J.P. Mitchell and T.L. Welsher, "Conductive Anodic Filament Growth in Printed Circuit Materials," Proceedings of the Printed Circuit World Convention II, pp. 80-93, 1981.

[9] G. W. Warren, P. Wynblatt and M. Zamanzadeh, "The role of electrochemical migration and moisture adsorption on the reliability of metallized ceramic substrates", J. Electron. Mater. Vol.18, No.2, 339-353, 1989. 
[10] B. S. Rudra and M. G. Pecht, "Assessing Time-to-Failure Due to Conductive Filament Formation in Multi-Layer Organic Laminates," Packaging and Manufacturing Techniques, Part B, Vol. 17, No. 3, pp. 269-276, 1994. 